\title{
On Blow-up Sets and Asymptotic Behavior of Interfaces of One Dimensional Quasilinear Degenerate Parabolic Equations
}

\author{
By \\ Ryuichi SuzUKI* \\ Dedicated to Professor Mutsuhide Matsumura on his 60 th birthday
}

\section{§1. Introduction}

In this paper we study the initial boundary value problem for a quasilinear degenerate parabolic equation of the form

$$
b(u)_{t}=u_{x x}+f(u) \quad \text { in } \quad x \in \Omega, t>0,
$$

with one dimensional open interval $\Omega \subset \mathbf{R}$ under the initial condition

$$
u(x, 0)=u_{0}(x) \quad \text { in } \quad x \in \Omega,
$$

together with one of the following three types of boundary conditions:

(a) the Dirichlet boundary conditions with $\Omega=(0, L)$

$$
u(0, t)=u(L, t)=0 \quad \text { in } \quad t>0
$$

(b) the Neumann boundary conditions with $\Omega=(0, L)$

$$
u_{x}(0, t)=u_{x}(L, t)=0 \quad \text { in } \quad t>0
$$

(c) the Cauchy problem, namely,

$$
\Omega=\mathbf{R} .
$$

We assume the following conditions on $b(u), f(u)$ and $u_{0}(x)$, respectively:

Communicated by T. Kawai, September 4, 1989. Revised September 21, 1990. 1991 Mathematics Subject Classification: 35K55.

* Tokyo Metropolitan College of Aeronautical Engineering, Arakawa-ku, Tokyo, 116, Japan. 
(A1) $b(u) \in C([0, \infty)) \cap C^{\infty}((0, \infty)), b^{-1}(v) \in C^{1}([0, \infty)), \lim _{u \rightarrow \infty} b(u)=\infty, b(0)$ $=0, b(u) \geq 0$ for $u \geq 0$ and $b^{\prime}(u)>0 b^{\prime \prime}(u) \leq 0$ for $u>0$ where $u=b^{-1}(v)$ is the inverse function of $v=b(u)$; $u>0$;

(A2) $f(u) \in C([0, \infty)) \cap C^{\infty}((0, \infty)), f\left(b^{-1}(v)\right) \in C^{1}([0, \infty))$ and $f(u)>0$ for

$$
u_{0}(x) \in B(\bar{\Omega}) \quad \text { and } \quad u_{0}(x) \geq 0 \quad \text { for } \quad x \in \bar{\Omega}
$$

where $B(k)$ is the set of all bounded continuous functions on a closed subset $K$ of R.

In the case of the Dirichlet problem, we assume in addition the following compatibility condition:

$$
u_{0}(0)=u_{0}(L)=0 .
$$

Furthermore, we assume the following condition so that weak solutions of (1.1) may blow up in a finite time:

$$
\int_{1}^{\infty} \frac{b^{\prime}(\xi)}{f(\xi)} d \xi<\infty
$$

The equation (1.1) is called a porous media type equation and it represents the process of thermal diffusion in a nonlinear continuous medium with the emission of thermal energy. And $u(x, t)$ represents a temperature and $f(u)$ represents a heat source.

Remark 1.1. Assumptions (A1), (A2) and (A4) are satisfied if, for example, the equation (1.1) is

$$
\left(u^{1 / m}\right)_{t}=u_{x x}+u^{p / m} \quad(p>1, m \geq 1) .
$$

Let us put $Q_{\tau}=\Omega \times(0, \tau)$. We know that if $\tau>0$ is small enough, there exists a unique non-negative weak solution $u(x, t)$ of $(1.1)(1.2)$ (1.3abc) (see e.q., [2], [3], [12] and [13]). The definition of "weak" solutions will be given below in Section 2.

Now let us put

$$
T=\sup \left\{\tau \mid u(x, t) \text { exists in } Q_{\tau}=\Omega \times(0, \tau)\right\} .
$$

If $u(x, t)$ does not exist globally in time, namely,

$$
0<T<\infty \text {, }
$$

then we call this solution a blow-up weak solution and we call $T$ a blow-up time. The local existence theorem implies

$$
\lim _{t \uparrow T} \sup \{u(x, t) \mid x \in \Omega\}=\infty .
$$


For studies on blow-up or non-blow-up of solutions, see references [6], [8], [9] and [10].

By a blow-up point of a blow-up weak solution $u(x, t)$ we mean a point $x \in \bar{\Omega} \cup\{\infty\} \cup\{-\infty\}$ such that there is a sequence $\left\{x_{n}, t_{n}\right\} \subset \bar{\Omega} \times(0, T)$ satisfying

$$
t_{n} \uparrow T, x_{n} \longrightarrow x \text { and } u\left(x_{n}, t_{n}\right) \longrightarrow \infty \text { as } n \longrightarrow \infty \text {. }
$$

Also we call the set of all blow-up points a blow-up set.

From the definition, we see that the blow-up set is a closed subset in $\mathbf{R} U$ $\{\infty\} \cup\{-\infty\}$. We shall study the shape of the blow-up set of each blow-up solution to (1.1)(1.2)(1.3abc) and furthermore, in the case of the Cauchy problem (1.1)(1.2)(1.3c) we shall study the asymptotic behavior of an interface of each blow-up solution of this problem near the blow-up time $t=T$.

In the case of the Dirichlet or Neumann problem, we shall show that if $f(u)$ grows more rapidly than $u$ (see (A5)), then the blow-up set of a blow-up solution is finite (Theorem 5.1). In the semilinear case $b(\xi)=\xi$, this result has already been obtained by Chen-Matano [4] and our methods are based on theirs. Note that we have to add some technical conditions on the initial data $u_{0}(x)$ (see (A6) and (A7)). On the other hand, we do not use the analyticity condition on $u_{0}(x)$ and $f(u)$ which is required in the case of the Dirichlet problem and in case $f(0)$ $>0$.

In the case of the Cauchy problem, we assume that the initial data $u_{0}(x)$ has a compact support $[0, L]$ (see (A8)). If we add some assumptions on $b(u)$ and $f(u)$ (see (A9)(A10)(A11)), we can obtain a finite propagation of the interface of the blow-up solution $u(x, t)$ in $t<T$. We are interested in the behavior of the interface near the blow-up time $t=T$ as well as the shape of the blow-up set.

We consider the following two cases:

(I) $f(u)$ grows more rapidly than $u$ (see (A5)).

(II) $f(u)$ grows more slowly than $u$ (see (A12)).

If (I) holds, we obtain that the blow-up set $S\left(u_{0}\right)$ is contained in $[0, L]$ and the interface stays bounded as $t \uparrow T$. Furthermore, if the technical condition (A7) is added on $u_{0}(x)$, then $S\left(u_{0}\right)$ becomes a finite set (Theorem 6.2 (i)). On the other hand, if (II) holds, we obtain that the blow-up set $S\left(u_{0}\right)$ is equal to $\mathbf{R} \cup\{\infty\} \cup$ $\{-\infty\}$ and consequently the interface propagates to the infinity as $t \uparrow T$ (Theorem 6.2 (ii)).

To prove these results, we can use the finite propagation property of the interface for (I) and (II). We can also use the non-blow-up result for the Dirichlet problem due to Imai-Mochizuki [8] for (II) only. In order to prove results for (I) we can also use the methods developed Friedman-McLeod [5] and Chen-Matano [4] for the semilinear problem. Note here that [8] studied the initial-boundary value problem (1.1)(1.2)(1.3ab) and asserted that the above two 
conditions (I) and (II) on $f(u)$ bring on the completely different blow-up situations.

Similar results to Theorem 6.3 were already obtained in [6] and [7] for the special equation (1.4) with $m>1$. However, the proof in $[6,7]$ strongly depends on the equation and it seems difficult to apply it directly to our general quasilinear equation.

The paper is structured as follows: In Section 2, we state the definition of weak solutions of (1.1)(1.2)(1.3abc) and we state some lemmas used throughout this paper. In Section 3, we also state some lemmas which will be directly used in our blow-up problems. In Section 4, we shall show that the blow-up set becomes finite under some special conditions on the blow-up solution $u(x, t)$. In Section 5 we consider the Dirichlet or Neumann problem using the results in Section 4 and in Section 6 we consider the Cauchy problem.

Acknowledgment. The author wishes to express his gratitude to Professor K. Mochizuki for his valuable suggestions and helpful encouragement. The author is also grateful to Professors M. Matsumura and Y. Shibata for their kind advice.

\section{§2. Definitions and Preliminaries}

In this section, we assume (A1)-(A4).

Definition 2.1. Let $\Omega$ be an open interval in $\mathbf{R}$ and let $T>0$. A function $u(x, t)$ defined in $Q_{T}=\Omega \times(0, T)$ is called a weak solution of (1.1), if:

1) $u(x, t) \in B\left(\bar{\Omega} \times\left[0, T^{\prime}\right]\right)$ for each $T^{\prime} \in(0, T)$, and $u(x, t) \geq 0$ for $(x, t) \in Q_{T}$;

2) For any $T^{\prime} \in(0, T)$ and any bounded open interval $\Omega^{\prime}=(\alpha, \beta)$ in $\Omega$, the identity

$$
\begin{aligned}
\int_{\Omega^{\prime}} b\left(u\left(x, T^{\prime}\right)\right) \varphi\left(x, T^{\prime}\right) d x-\int_{\Omega^{\prime}} b(u(x, 0)) \varphi(x, 0) d x & \\
-\int_{0}^{T^{\prime}} \int_{\Omega^{\prime}} b(u(x, t)) \varphi_{t} d x d t & =\int_{0}^{T^{\prime}} \int_{\Omega^{\prime}} u \varphi_{x x}(x, t) d x d t-\left.\int_{0}^{T^{\prime}} u \frac{\partial \varphi}{\partial x} d t\right|_{x=\beta} ^{x=\alpha} \\
& +\int_{0}^{T^{\prime}} \int_{\Omega^{\prime}} f(u) \varphi(x, t) d x d t
\end{aligned}
$$

holds for any test function $\varphi(x, t) \in C^{2,1}\left(\bar{\Omega}^{\prime} \times[0, T)\right)$ satisfying $\varphi(\alpha, t)=\varphi(\beta, t)$ $=0$ for $t \in(0, T)$ and $\varphi(x, t) \geq 0$ for $t \in(0, T)$.

A function $u(x, t)$ defined in $Q_{T}=\Omega \times(0, T)$ is called a weak super-(sub-) solution of (1.1), if $u(x, t)$ satisfies 1$)$ and 2) with equality replaced by $\geq(\leq)$.

A function $u(x, t)$ defined in $(0, L) \times(0, T)$ is called a weak solution of the Dirichlet problem (1.1)(1.2)(1.3a), if $u(x, t)$ is a weak solution of $(1.1)$ in $(0, L)$ 
$\times(0, T)$ and, if $u(0, t)=u(L, t)=0$ in $t \in(0, T)$ and $u(x, 0)=u_{0}(x)$ in $x \in(0, L)$.

A function $u(x, t)$ defined in $(0, L) \times(0, T)$ is called a weak solution of the Neumann problem (1.1)(1.2)(1.3b), if $u(x, t)$ is a weak solution of $(1.1)$ in $(0, L)$ $\times(0, T)$ and, if $u(x, t)$ satisfies 1$)$ and 2$)$ with $\varphi(x, t)$ replaced by $\varphi(x, t) \in C^{2,1}$ $\left(\bar{\Omega}^{\prime} \times[0, T)\right)$ satisfying $\varphi(x, t)=0$ for $(x, t) \in(\{\alpha, \beta\} \backslash\{0, L\}) \times[0, T)$ and $\varphi_{x}(x, t)$ $=0$ for $(x, t) \in\{\alpha, \beta\} \cap\{0, L\} \times(0, T)$, and if $u(x, 0)=u_{0}(x)$ for $x \in \Omega$.

A function $u(x, t)$ defined in $(0, L) \times(0, T)$ is called a weak super-(sub-) solution of the Neumann problem (1.1) (1.3b), if $u(x, t)$ satisfies 1) and 2) with equality replaced by $\geq(\leq)$ and with $\varphi(x, t)$ replaced by $\varphi(x, t) \in C^{2,1}(\bar{\Omega}$ $\times[0, T))$ satisfying $\varphi(x, t)=0$ for $(x, t) \in(\{\alpha, \beta\} \backslash\{0, L\}) \times[0, T)$ and $\varphi_{x}(x, t)$ $=0$ for $(x, t) \in\{\alpha, \beta\} \cap\{0, L\} \times(0, T)$.

A function $u(x, t)$ defined in $\mathbf{R} \times(0, T)$ is called a weak solution of the Cauchy problem (1.1)(1.2)(1.3c), if $u(x, t)$ is a weak solution of (1.1) in $\mathbf{R} \times(0, T)$ and, if $u(x, 0)=u_{0}(x)$ for $x \in \mathbf{R}$.

Lemma 2.2 (the comparison theorem). Assume (A1)-(A4). Let $\Omega$ be an open interval in $\mathbf{R}$. Then, the two following results hold:

(i) Suppose that $u(x, t)$ is a super-solution of (1.1) in $Q_{T}=\Omega \times(0, T)$ and $v(x, t)$ is a sub-solution of $(1.1)$ in $Q_{T}=\Omega \times(0, T)$. Then, if $u(x, t) \geq v(x, t)$ for $(x, t) \in \partial \Omega \times(0, T)$ and if $u(x, 0) \geq v(x, 0)$ for $x \in \Omega, u(x, t) \geq v(x, t)$ for all $(x, t) \in \Omega \times(0, T)$.

(ii) Suppose that $u(x, t)$ is a super-solution of the Neumann problem $(1.1)(1.3 b)$ in $Q_{T}=\Omega \times(0, T)$ and $v(x, t)$ is a sub-solution of the Neumann problem (1.1)(1.3b). Then, if $u(x, 0) \geq v(x, 0)$ for $x \in(0, L), u(x, t) \geq v(x, t)$ for all $(x, t) \in \Omega \times(0, T)$.

Proof. See Aronson-Grandall-Peletier [2] and Bertsch-Kersner-Peletier [3].

Finally we show the following lemma:

Lemma 2.3. Assume (A1)-(A4). Let $u(x, t)$ be a weak solution of (1.1) in $Q_{T}$. Then, if there exists a point $(x, t) \in Q_{T}$ such that $u\left(x_{0}, t_{0}\right)>0, u(x, t)$ is a $C^{\infty}$ function in a neighborhood of $\left(x_{0}, t_{0}\right)$ and

$$
u\left(x_{0}, t\right) \geq \eta(t)>0 \quad t \in\left(t_{0}, T\right)
$$

where $\eta(t)$ is a solution of an ordinary differential equation $\eta^{\prime}=-\lambda \eta / b^{\prime}(\eta)$ for some positive constant $\lambda$.

Proof. We only show (2.1). By the fact that $u\left(x_{0}, t_{0}\right)>0$, there exist $\delta>0$ and $\alpha>0$ such that

$$
u(x, t) \geq \alpha>0 \text { for }\left|x-x_{0}\right|<\delta \text { and }\left|t-t_{0}\right|<\delta .
$$




$$
v(x, t)=g(x) \eta(t)
$$

where

$$
g(x)=\sin \left(\left\{x-\left(x_{0}-\delta\right)\right\} \pi / 2 \delta\right)
$$

and $\eta(t)$ satisfies a differential equation

$$
\eta^{\prime}=-\lambda \frac{\eta}{b^{\prime}(\eta)}
$$

with $\eta\left(t_{0}\right)=\alpha$ and $\lambda=(\pi / 2 \delta)^{2}$.

We shall show

$$
u(x, t) \geq v(x, t) \text { for all }(x, t) \in\left[x_{0}-\delta, x_{0}+\delta\right] \times\left[t_{0}, T\right) .
$$

First, $\eta$ can be represented explicity by

$$
\eta(t)=W^{-1}\left(W(\alpha)-\lambda\left(t-t_{0}\right)\right)
$$

where

$$
W(\eta)=\int_{1}^{\eta} \frac{b^{\prime}(\xi)}{\xi} d \xi, \quad \eta>0
$$

and $W^{-1}$ is the inverse function of $W$. Noting that $W(\eta)$ is an increasing function and $W(\eta) \rightarrow-\infty$ as $\eta \downarrow 0$, we have that $\eta(t)>0$ in $t \in\left(t_{0}, \infty\right)$ and $\eta(t) \downarrow 0$ as $t \rightarrow \infty$. Since $1 / b^{\prime}(\eta)$ is an increasing function, a simple calculation shows that

$$
b(v)_{t} \leq v_{x x}+f(v) \quad \text { for } \quad(x, t) \in\left(x_{0}-\delta, x_{0}+\delta\right) \times\left(t_{0}, T\right) .
$$

Thus, we see that $v(x, t)$ is a sub-solution of (1.1) in $\left(x_{0}-\delta, x_{0}+\delta\right) \times\left(t_{0}, T\right)$ and that

$$
v\left(x_{0} \pm \delta, t\right)=0 \leq u\left(x_{0} \pm \delta, t\right) \quad \text { for } \quad t \in\left(t_{0}, T\right)
$$

and

$$
v\left(x, t_{0}\right)=\alpha g(x) \leq u\left(x, t_{0}\right) \quad \text { for } \quad x \in\left[x_{0}-\delta, x_{0}+\delta\right] .
$$

Applying the comparison theorem to $u(x, t)$ and $v(x, t)$, we obtain that $u(x, t) \geq v(x, t),(x, t) \in\left[x_{0}-\delta, x_{0}+\delta\right] \times\left[t_{0}, T\right) . \quad$ This proof is complete.

\section{§3. Fundamental Lemmas}

In this section, we assume (A1)-(A4). We state some fundamental lemmas used after this section.

Lemma 3.1. Assume (A1)-(A4). Let $\Omega=(a, d)$ be a bounded open interval 
and, let $u(x, t)$ be a weak solution of (1.1) in $Q_{T}=\Omega \times(0, T)$. Then, the following two results hold:

(i) Suppose that $u(a, t)>0$ for $t \in[0, T), \quad u(a, 0)>u(d, 0) \geq 0$ and $\sigma_{c} u(x, t) \geq u(x, t)$ for $(x, t) \in[c, d] \times[0, T)$ where $\sigma_{c} u(x, t)=u(2 c-x, t)$ with $c$ $=(a+d) / 2$. Then, if there exists $t_{0} \in(0, T)$ such that $u(x, t)>0$ for $x \in[a, c]$,

$$
u_{x}\left(c, t_{0}\right)<0
$$

(ii) Suppose that $u(d, t)>0$ for $t \in[0, T), u(d, 0)>u(a, 0) \geq 0$ and $\sigma_{c} u(x, t) \geq u(x, t)$ for $(x, t) \in[a, c] \times[0, T)$ where $\sigma_{c} u(x, t)$ is as above. Then, if there exists $t_{0} \in(0, T)$ such that $u\left(x, t_{0}\right)>0$ for $x \in[c, d]$,

$$
u_{x}\left(c, t_{0}\right)>0 \text {. }
$$

Proof. We shall only show (3.1).

First, we show that

$$
\sigma_{c} u\left(x, t_{0}\right)>u\left(x, t_{0}\right) \quad \text { for } \quad c<x \leq d .
$$

Assume that $\sigma_{c} u\left(x_{0}, t_{0}\right)-u\left(x_{0}, t_{0}\right)=0$ for some $x_{0} \in(c, d)$. Set $w=v-u$ where $v=\sigma_{c} u$. Then we see that $w(x, t)$ satisfies a linear parabolic equation

$$
w_{t}=\frac{1}{b^{\prime}(u)}\left\{w_{x x}+\left\{\bar{f}-\bar{b}^{\prime} v_{t}\right\} w\right\}
$$

in $\quad(x, t) \quad$ satisfying $\quad w(x, t)>0, \quad$ where $\quad \bar{\varphi}=\bar{\varphi}(v, u)=\int_{0}^{1} \bar{\varphi}^{\prime}(\theta v+(1-\theta) u)$

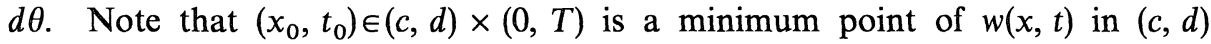
$\times[0, T)$. Applying the maximum principle to $w(x, t)$, we obtain that $w\left(x, t_{0}\right)$ $=0$ in $x \times[c, d]$, which implies $w(d, t)=0$ in $t \in\left[0, t_{0}\right]$. Namely $w(d, 0)$ $=u(a, 0)-u(d, 0)=0$. However this contradicts the assumption of Lemma 3.1.

Next we show (3.1). Note that $u(x, t)>0$ in the neighborhood of $t=t_{0}$ and $x \in[a, c]$. Then, by the same methods by which we demonstrated to show (3.3) we have that $w(x, t)>0$ in $(x, t) \in(c, d] \times\left(t_{0}-\delta, t_{0}+\delta\right)$ for some $\delta>0$. Applying the maximum principle to $w(x, t)$ and using the fact that $w(c, t)=0$, we have that $w_{x}(c, t)>0$ for $t \in\left(t_{0}-\delta, t_{0}+\delta\right)$. Namely $u_{x}(c, t)<0$ for $t \in\left(t_{0}\right.$ $\left.-\delta, t_{0}+\delta\right)$. This is a proof of (3.1).

Next, we further assume the following condition:

(A5) There exists a $C^{\infty}$-function $F:[0, \infty) \rightarrow[0, \infty)$ such that

(i) $F(u)>0, F^{\prime}(u) \geq 0, F^{\prime \prime}(u) \geq 0$, for $u>0$;

(ii) there exist $c>0$ and $M_{0}>0$ such that,

$$
f^{\prime} F-f F^{\prime} \geq c\left(F^{\prime} F-\frac{b^{\prime \prime}}{b^{\prime}} F^{2}\right) \quad \text { for } \quad u>M_{0}
$$


(iii)

$$
\int_{1}^{\infty} \frac{d u}{F(u)}<\infty
$$

Remark 3.2. If $p / m>1$, equation (1.4) satisfies this condition.

Lemma 3.3 ( $c f$. Friedman-McLeod [5], Chen-Matano [4]). Assume (A1)(A5). Let $\Omega=(a, b)$ be a bounded open interval and let $u(x, t)$ be a positive weak solution of (1.1) in $Q_{T}=\Omega \times(0, T)$. Furthermore suppose that

$$
u_{x}(x, t)>0\left[\text { or } u_{x}(x, t)<0\right] \text { in }(x, t) \in[c-\delta, c+\delta] \times(\tau, T),
$$

for some $c \in(a, b)$ and $\delta>0$ with $(c-\delta, c+\delta) \subset(a, b)$ and some $\tau \in(0, T)$. Then there are no blow-up points in $(c-\delta, c+\delta)$.

Proof. We shall show this lemma in case

$$
u_{x}(x, t)>0 \quad \text { in } \quad(x, t) \in(c-\delta, c+\delta) \times(\tau, T) .
$$

We give an indirect proof. Assume that $x_{0} \in(c-\delta, c+\delta)$ is a blow-up point of $u(x, t)$. Then we see that

$$
\lim _{t \uparrow T} u(x, t)=\infty \quad \text { for } \quad x \in\left(x_{0}, c+\delta\right) .
$$

In fact, let $d \in\left(x_{0}, c+\delta\right)$ be fixed. Since $x_{0}$ is a blow-up point, there exist sequences $\left\{x_{k}\right\} \subset(c-\delta, d)$ and $\left\{t_{k}\right\} \subset(t, T)$ such that $x_{k} \rightarrow x_{0}, t_{k} \rightarrow T$ and $u\left(x_{k}, t_{k}\right) \uparrow \infty$ as $k \rightarrow \infty$. By (3.8), we obtain

$$
u\left(x, t_{k}\right)>u\left(x_{k}, t_{k}\right) \quad \text { for } \quad d \leq x \leq c+\delta .
$$

Hence, by Lemma 2.3 we have

$$
u(x, t) \geq \eta_{k}(t) \sin \left(\frac{x-d}{c+\delta-d} \pi\right) \quad \text { for } \quad x \in[d, c+\delta], T>t \geq t_{k} .
$$

Here $\eta_{k}(t)=W^{-1}\left(W(\alpha)-\lambda\left(t-t_{k}\right)\right)$ and $W(\eta)=\int_{1}^{\eta} \frac{b^{\prime}(\xi)}{\xi} d \xi$ with $\alpha=u\left(x_{k}, t_{k}\right)$ and $\lambda=(\pi /(c+w-d))^{2}$. Since $W(\eta)$ is a monotone increasing function, we have

$$
\eta_{k}(t) \geq W^{-1}\left(W\left(u\left(x_{k}, t_{k}\right)\right)-\lambda\left(T-t_{k}\right)\right) \quad \text { in } t \in\left[t_{k}, T\right) .
$$

Noting that $\lim _{k \rightarrow \infty}\left\{W\left(u\left(x_{k}, t_{k}\right)-\lambda\left(T-t_{k}\right)\right\}=\lim _{\eta \uparrow \infty} w(\eta)\right.$, we obtain

$$
\min _{t \in\left[t_{k}, T\right)} \eta_{k}(t) \geq W^{-1}\left(W\left(u\left(x_{k}, t_{k}\right)\right)-\lambda\left(T-t_{k}\right)\right) \rightarrow \infty \text { as } k \rightarrow \infty .
$$

This and (3.11) show (3.9) since $d \in\left(x_{0}, c+\delta\right)$ is chosen arbitrarily.

Choose $d \in\left(x_{0}, c+d\right)$ again and set 


$$
J=u_{x}-\varepsilon \rho(x) F(u(x, t)), \quad(x, t) \in Q=(d, c+\delta) \times\left(t_{1}, T\right)
$$

and

$$
\rho(x)=\sin \frac{\pi(x-d)}{c+\delta-d}
$$

where $\varepsilon>0$ and $t_{1} \in(\tau, T)$. Noting the assumptions (A1), (A4), (A5) and (3.7), and assuming that $\varepsilon$ is sufficiently small and $t_{1}$ is sufficiently close $t=T$, we have

$$
\begin{gathered}
\left(b^{\prime} J\right)_{t}-J_{x x} \geq B(x, t) J+C(x, t) J, \quad(x, t) \in Q \\
J(d, t)>0, \quad J(c+\delta, t)>0, \quad t_{1} \leq t<T \\
J\left(x, t_{1}\right)>0, \quad d \leq x \leq c+\delta .
\end{gathered}
$$

(cf. Chen-Matano [4] and Imai-Mochizuki [8]). Applying the maximum principle to (3.14) (3.15) and (3.16), we obtain $J(x, t)>0$ for $(x, t) \in Q$, or

$$
\frac{u_{x}(x, t)}{F(u(x, t))}>\varepsilon \rho(x) \quad \text { in } \quad(x, t) \in Q .
$$

Integrating this inequality over $d \leq x \leq c+\delta$ yields

$$
\int_{u(d, t)}^{u(c+\delta, t)} \frac{d u}{F(u)}>\varepsilon \int_{d}^{c+\delta} \rho(x) d x \quad \text { in } \quad t_{1}<t<T .
$$

The right-hand side of (3.18) is a positive constant, while the left-hand side tends to zero as $t \uparrow T$ by virtue of condition (A5)(iii) and (3.9). This contradiction shows that $x_{0}$ is a not blow-up point of $u(x, t)$. Then proof is complete.

Finally we show

Lemma 3.4. Let $u(x, t)$ be as in Lemma 3.3. Then $u(x, t)$ can be extend to a $C^{2,1}$-function in $(c-\delta, c+\delta) \times(0, T]$. Moreover if we represent this $C^{2,1}$ function as $u(x, t)$ again, then

$$
u_{x}(x, T)>0\left[\text { or } u_{x}(x, T)<0\right] \quad \text { in } \quad x \in(c-\delta, c+\delta) .
$$

Proof. We shall show this lemma in case $u_{x}>0$. By Lemma 2.3 and Lemma 3.3, for any $c-\delta<d_{1}<d_{2}<c+\delta$, there exists $M^{\prime}=M^{\prime}\left(d_{1}, d_{2}\right)>0$ such that

$$
\frac{1}{M^{\prime}} \leq u(x, t) \leq M^{\prime} \quad \text { for } \quad(x, t) \in\left[d_{1}, d_{2}\right] \times[\tau, T) .
$$

Therefore we can easily extend $u(x, t)$ to $C^{2,1}$-function $u(x, t)$ in $(c-\delta, c+\delta)$ $\times[\tau, T]$ by means of standard $L^{p}$ and Schauder's estimates (cf. Chen-Matano [4]). 
Choose $d \in(c-\delta, c+\delta)$ arbitrarily and choose $l>0$ such that $[d-l, d+l]$ $\subset(c-\delta, c+\delta)$. Let us consider the following initial boundary value problem:

$$
\begin{cases}\dot{b}(v)_{t}=v_{x x}+f(v) & \text { in } \quad x \in(d-l, d+l), t>\tau, \\ v(x, \tau)=u(x, \tau) & \text { in } \quad x \in(d-l, d+l), \\ v(d \pm l, t)=\eta_{ \pm}(t) & \text { in } \quad t>\tau,\end{cases}
$$

where $\eta_{ \pm}(t)$ are continuous functions on $[\tau, \infty)$ and satisfies that $\eta_{ \pm}(t)=$ $u(a \pm l, t)$ for $t \in[\tau, T)$ and $\eta_{-}(t) \leq \eta_{+}(t)$ for $t \in[\tau, \infty)$. Then, there exists $T^{\prime}>0$ such that a solution $v(x, t)$ of (3.20) exists in $(d-l, d+l) \times\left(\tau, T+T^{\prime}\right)$ by the existence theorem, and the uniqueness theorem implies

$$
v(x, t)=u(x, t) \quad \text { in } \quad(x, t) \in(d-l, d+l) \times[\tau, T] .
$$

We compare $\sigma_{d} v(x, t)$ and $v(x, t)$ in $[d-l, d] \times\left[\tau, T+T^{\prime}\right)$. We can see easily that $\sigma_{d} v(x, \tau) \geq v(x, \tau)$ for $x \in[d-l, d], \sigma_{d} v(d, t)=v(d, t)$ for $t \in\left[\tau, T+T^{\prime}\right)$ and $\sigma_{d} v(d-l, t)-v(d-l, t)=\eta_{+}(t)-\eta_{-}(t) \geq 0$ for $t \in\left[\tau, T+T^{\prime}\right)$. Noting $\sigma_{d} v$ and $v$ are solutions of (1.1) and applying the comparsion theorem, we obtain

$$
\sigma_{d} v(x, t) \geq v(x, t) \quad \text { in } \quad(x, t) \in[d-l, d] \times\left[\tau, T+T^{\prime}\right) .
$$

Then it follows from Lemma 3.1 that

$$
u_{x}(d, t)>0 \quad \text { for } \quad t \in\left(\tau, T+T^{\prime}\right)
$$

so $u_{x}(d, T)>0$. Since $d \in(c-\delta, c+\delta)$ is chosen arbitrarily, we obtain (3.19).

\section{§4. Key Lemma}

In this section, we assume (A1)-(A5) and prove the following key lemma for the case of a bounded domain:

Lemma 4.1. Assume (A1)-(A5). Let $u(x, t)$ be a positive weak solution of (1.1) in $Q_{T}=\Omega \times(0, T)=(0, L) \times(0, T)$ and let $0<a_{1}<a_{1}<L$. Suppose that for any $t_{0} \in(0, T)$ there exists $\delta=\delta\left(t_{0}\right)>0$ such that

$$
u_{x}(x, t)>0\left[\text { or } u_{x}(x, t)<0\right], \quad(x, t) \in\left[a_{1}-\delta, a_{1}+\delta\right] \times\left(t_{0}, T\right)
$$

and

$$
u_{x}(x, t)<0\left[\text { or } u_{x}(x, t)>0\right], \quad(x, t) \in\left[a_{2}-\delta, a_{2}+\delta\right] \times\left(t_{0}, T\right)
$$

where $\left[a_{i}-\delta, a_{i}+\delta\right] \subset \Omega \quad(i=1,2)$ and $\left[a_{1}-\delta, a_{1}+\delta\right] \cap\left[a_{2}-\delta, a_{2}+\delta\right]$

$=\phi$. Then, the blow-up set of $u(x, t)$ is finite in $\left(a_{1}-\delta, a_{2}+\delta\right)$.

Remark 4.2. Consider the Dirichlet problem (1.1)(1.2)(1.3a) with $b(u)$ 
$=u$. Let $u(x, t)$ be a blow-up solution of the problem. Then, we see that $u(x, t)>0$ for $(x, t) \in(0, L) \times(0, T)$ and for any $\tau \in(0, T)$ there exists $\delta=\delta(\tau)>0$ such that $u_{x}(x, t)>0, \quad(x, t) \in(0, \delta) \times(\tau, T)$ and $u_{x}(x, t)<0,(x, t) \in(L-\delta, L)$ $\times(\tau, T)$ (see Friedman-McLeod [5]). Using Lemma 4.1 and the fact that the blow-up set of $u(x, t)$ is contained in $(0, L)$ (Friedman-McLeod [5]), we obtain that the blow-up set of $u(x, t)$ is a finite set. Then, we do not use the analyticity condition on a initial data $u_{0}(x)$ and a heat source $f(u)$ which is required in Chen-Matano [4] in case $f(0)>0$.

We need some notations and preliminary lemmas (see Chen-Matano [4] and Angenent [1]).

Notation 4.3. Let $w(x)$ be a continuous real value function on $K$ where $K$ is $S^{1}=\mathbf{R} / \mathbb{Z}$ or a bounded closed interval in $\mathbf{R}$. We define the nodal number of $w$ by

$$
v(w)=\text { the number of points } x \in K \text { with } w(x)=0 .
$$

This defines a functional $v: C(K) \rightarrow N \cup\{0\} \cup\{\infty\}$.

Definition 4.4. We say that $w \in C^{1}(K)$ poses only simple zeroes if $w^{\prime}(x) \neq 0$ for any $x \in K$ such that $w(x)=0$. The set of all such functions is denoted by $\Sigma(K)$.

Lemma 4.5 (Angenent). Let $p(x, t), q(x, t)$ and $r(x, t)$ be locally bounded continuous functions on $S^{1} \times\left(t_{0}, T\right)$ with $p_{x x}, p_{x t}, p_{t t}, p_{x}, p_{t}, q_{x}, q_{t}$, all locally bounded continuous. Furthermore, let $p(x, t)>0$ and let $w(x, t)$ be a classical solution of

$$
w_{t}=p(x, t) w_{x x}+q(x, t) w_{x}+r(x, t) w,(x, t) \in S^{1} \times\left(t_{0}, T\right) .
$$

Assume that $w$ is not identically equal to zero. Then

(i) $v(w(\cdot, t))$ is finite for any $t \in\left(t_{0}, T\right)$ and is monotone nonincrasing in $t$;

(ii) there exists a strictly decreasing sequence of points $\left\{t_{k}\right\} \subset\left(t_{0}, T\right)$ such that $\left\{t_{k}\right\} \downarrow t_{0}$ and $w(\cdot, t) \in \Sigma\left(S^{1}\right)$ for any $t \in\left(t_{0}, T\right) \backslash\left\{t_{k}\right\}$.

Lemma 4.6 (Angenent). The assertions of Lemma 4.5 hold with $S^{1}$ replaced by a closed interval $[a, b]$ in $\mathbf{R}$, if we assume in addition that $w(a, t) \neq 0$ and $w(b, t) \neq 0$ for any $t \in\left(t_{0}, T\right)$.

Remark 4.7. Lemma 4.5 and Lemma 4.6 follow immediately from the next lemma due to Angenent [1]:

Lemma 4.8 ([1]). Under the assumption of Lemma 4.1 or of Lemma 4.2, we have

(i) $v(w(\cdot, t))$ is finite for $t \in\left(t_{0}, T\right)$.

(ii) If $\left(x_{0}, t_{1}\right)$ is multiple zero of $w$, then $v\left(w\left(\cdot, t_{2}\right)\right)>v\left(w\left(\cdot, t_{3}\right)\right)$ for all $t_{2}$ 
$<t_{1}<t_{3}<T$

With Lemma 4.6 we can now prove Lemma 4.1.

Proof of Lemma 4.1 (cf. Chen-Matano). We note that the point of $\left(\mathrm{a}_{1}-\delta\left(t_{0}\right), a_{1}+\delta\left(t_{0}\right)\right) \cup\left(a_{2}-\delta\left(t_{0}\right), a_{2}+\delta\left(t_{0}\right)\right)$ is not a blow-up point.

By differentiating equation (1.1) with respect to $x$, we see that $w=u_{x}(x, t)$ satisfies a parabolic equation of the form (4.3) in $\left[a_{1}, a_{2}\right] \times\left(t_{0}, T\right)$. Therefore noting (4.1) and (4.2) and using Lemma 4.6 (ii), we can see the existence of $\tau \in\left(t_{0}, T\right)$ such that

$$
u_{x}(\cdot, t) \in \Sigma\left(\left[a_{1}, a_{2}\right]\right) \quad \text { for all } t \in[\tau, T) .
$$

Applying the implicit function theorem to $u_{x}$, we obtain $C^{1}$-curves $\xi_{1}, \xi_{2}, \cdots, \xi_{n}:[\tau, T) \rightarrow\left(a_{1}, a_{2}\right)$ such that

(i) $\xi_{1}(t)<\xi_{2}(t)<\cdots<\xi_{n}(t)$ for $t \in[\tau, T)$,

and

(ii) $\left\{x \in\left[a_{1}, a_{2}\right] \mid u_{x}(x, t)=0\right\}=\left\{\xi_{1}(t), \cdots, \xi_{n}(t)\right\}$

for each $t \in[\tau, T)$.

Let $S\left(u_{0}\right)$ be the blow-up set of $u(x, t)$. We shall show

$$
\lim _{t \uparrow T} \xi_{i}(t)=\alpha_{i} \text { exists for each } 1 \leq i \leq n
$$

and

$$
S\left(u_{0}\right) \cap\left(a_{1}, a_{2}\right) \subset\left\{\alpha_{1}, \alpha_{2}, \cdots, \alpha_{n}\right\}
$$

First, set $\alpha_{i}^{-}=\lim _{t \uparrow T} \inf \xi_{i}(t), \alpha_{i}^{+}=\lim \sup _{t \uparrow T} \xi_{i}(t)$ and $J_{i}=\left[\alpha_{i}^{-}, \alpha_{i}^{+}\right]$for each $1 \leq i \leq n$. Then, by (4.1) and (4.2) we have

$$
\bigcup_{i=1}^{n} J_{i} \subset\left(a_{1}, a_{2}\right)
$$

Moreover we obtain

$$
\left(a_{1}, a_{2}\right) \backslash \bigcup_{i=1}^{n} J_{i} \subset\left(a_{1}, a_{2}\right) \backslash S\left(u_{0}\right)
$$

In fact, choose a closed interval $[c, d] \subset\left(a_{1}, a_{2}\right) \backslash \bigcup_{i=1}^{n} J_{i}(c<d)$ arbitrarily. Then, there exists $t_{1} \in(\tau, T)$ such that $u_{x}(x, t) \neq 0$ does not change its sign in the rectangular region $[c, d] \times\left[t_{1}, T\right)$. It follows from Lemma 3.3 that $(c, d)$ $\subset\left(a_{1}, a_{2}\right) \backslash S\left(u_{0}\right)$. Since $[c, d] \subset\left(a_{1}, a_{2}\right) \backslash \bigcup_{i=1}^{n} J_{i}$ is chosen arbitrarily, (4.8) follows. 
Next we define the family $\left\{W_{i}\right\}$ of sets inductively as follows: For closed intervals $A_{1}=\left[c_{1}, d_{1}\right]$ and $A_{2}=\left[c_{2}, d_{2}\right]$, set $\left(A_{1}, A_{2}\right)=\left(\left(c_{1}+c_{2}\right) / 2,\left(d_{1}+d_{2}\right) / 2\right)$ and define $W_{j}(j=0,1, \cdots)$ inductively as

$$
\begin{aligned}
W_{0}=\left\{\left(a_{1}-\delta, a_{1}+\delta\right),\left(a_{2}-\delta, a_{2}+\delta\right)\right\} \\
W_{1}=\left\{A \mid A=\left(\bar{A}_{1}, \bar{A}_{2}\right), \quad A_{1}, A_{2} \in W_{0}\right\} \\
\ldots \ldots \ldots \ldots \ldots \ldots \ldots \ldots \ldots \ldots \ldots \ldots \ldots \ldots \ldots \\
W_{j+1}=\left\{A \mid A=\left(\bar{A}_{1}, \bar{A}_{2}\right), \quad A_{1}, A_{2} \in W_{j}\right\}
\end{aligned}
$$

Then, we see easily the following properties with $\tilde{W}_{j}=\bigcup_{A \in W_{j}} A$ :

$$
\begin{gathered}
W_{j} \subset W_{j+1} \quad \text { for } \quad j \geq 0, \\
\tilde{W}_{j} \subset\left(a_{1}-\delta, a_{2}+\delta\right) \quad \text { for each } j,
\end{gathered}
$$

and there exists $m \geq 1$ such that

$$
\left(a_{1}, a_{2}\right) \subset \bigcup_{j=0}^{m} \tilde{W}_{j}
$$

Hence, if we show

$$
\left(\bigcup_{i=1}^{n} \stackrel{\circ}{J}_{i}\right) \cap\left(\bigcup_{j=0}^{m} \tilde{W}_{j}\right)=\phi
$$

where $\stackrel{\circ}{K}$ is the set of all interior points of a subset $K$ in $\mathbf{R}$, then we have $\bigcup_{i=1}^{n} \stackrel{\circ}{J}_{i}$ $=\phi$ by (4.7), and therefore we obtain (4.5) and (4.6) by (4.8).

Let us show (4.12). Suppose that (4.12) does not hold. Then, by (4.9) there exists $j_{0}\left(1 \leq j_{0} \leq m\right)$ such that

$$
\begin{gathered}
\tilde{W}_{j_{0}-1} \cap\left(\bigcup_{i=1}^{n} \stackrel{\circ}{J}_{i}\right)=\phi \\
\tilde{W}_{j_{0}} \cap\left(\bigcup_{i=1}^{n} \stackrel{\circ}{J}_{i}\right) \neq \phi .
\end{gathered}
$$

Since $A$ is represented as $A=\left(\bar{A}_{1}, \bar{A}_{2}\right)$ for some $A_{1}, A_{2} \in W_{j_{0}-1}$, it follows from (4.8) and (4.13) that

$$
\left\{A_{1} \cup A_{2}\right\} \backslash\left\{\alpha \mid \alpha=\alpha_{i}^{-}=\alpha_{i}^{+}\right\} \subset\left(a_{1}, a_{2}\right) \backslash S\left(u_{0}\right) .
$$

On the other hand, by (4.14), there exists $i_{0}\left(1 \leq i_{0} \leq n\right)$ such that

$$
A \cap \stackrel{\circ}{J}_{i_{0}} \neq \phi \text {. }
$$


Noting that $A=\left(\bar{A}_{1}, \bar{A}_{2}\right)$, there exist $a \in A_{1} \backslash\left\{\alpha \mid \alpha=\alpha_{i}^{-}=\alpha_{i}^{+}\right\}$and a non-empty open interval $D \subset A_{2} \backslash\left\{\alpha \mid \alpha=\alpha_{i}^{-}=\alpha_{i}^{+}\right\}$such that

$$
(a, \bar{D})=\left\{\frac{a+x}{2} \mid x \in D\right\} \subset A \cap \stackrel{\circ}{J}_{i_{0}} .
$$

Hence, by (4.15) we have

$$
D \subset\left(a_{1}, a_{2}\right) \backslash S\left(u_{0}\right) .
$$

Noting that $D \subset A_{2} \backslash\left\{\alpha \mid \alpha=\alpha_{i}^{-}=\alpha_{i}^{+}\right\}$, there exist $t_{2} \in\left(t_{1}, T\right)$ and a non-empty closed interval $K \subset D$ such that $u_{x} \neq 0$ does not change its sign in $K$ $\times\left[t_{2}, T\right)$. Therefore, by Lemma 3.3, we can extend $u(x, t)$ to a $C^{2,1}$-function in $\stackrel{\circ}{K} \times\left[t_{2}, T\right]$ uniquely and we may see that $u_{x}(x, T)>0$ for all $x \in \stackrel{\circ}{K}$. Since the $\lim _{t \uparrow T} u(a, t)=u(a, T)$ also exists by Lemma 3.3, we can see the existence of $x \in \stackrel{\circ}{K}$ such that $u\left(x_{0}, T\right) \neq u(a, T)$. Hence there exists $t_{2}^{\prime} \in\left[t_{2}, T\right)$ such that

$$
u\left(x_{0}, t\right) \neq u(a, t) \quad \text { for all } t \in\left[t_{2}^{\prime}, T\right) .
$$

Set $w(x, t)=\sigma_{b} u(x, t)-u(x, t)$ where $\sigma_{b} u(x, t)=u(2 b-x, t)$ with $b=\left(x_{0}\right.$ $+a) / 2$. Assume that $x_{0}<a$ for convenience. Then we can see that $w(x, t)$ is a solution of a linear parabolic equation of the form (4.3) in $\left[x_{0}, a\right]$ $\times\left(t_{2}^{\prime}, T\right)$. Moreover, it follows from (4.19) that

$$
\left\{\begin{array}{l}
w\left(x_{0}, t\right)=\sigma_{b} u\left(x_{0}, t\right)-u\left(x_{0}, t\right)=u(a, t)-u\left(x_{0}, t\right) \neq 0, \quad t \in\left(t_{2}^{\prime}, T\right) \\
w(a, t)=u\left(x_{0}, t\right)-\sigma_{b} u\left(x_{0}, t\right) \neq 0, \quad t \in\left(t_{2}^{\prime}, T\right)
\end{array}\right.
$$

Applying Lemma 4.6 to $w(x, t)$ in $\left[x_{0}, a\right] \times\left(t_{2}^{\prime}, T\right)$, we see that there exists $t_{3} \in\left(t_{2}^{\prime}, T\right)$ such that $w(\cdot, t) \in \Sigma\left(\left[x_{0}, a\right]\right)$ for all $t \in\left[t_{3}, T\right)$. Hence, noting $w(b, t)$ $=u(b, t)-u(b, t)=0$, we have $w_{x}(b, t) \neq 0$ for $t \in\left[t_{3}, T\right)$, namely,

$$
u_{x}(b, t) \neq 0 \quad \text { for all } t \in\left[t_{3}, T\right) \text {. }
$$

On the other hand, since $b \in \stackrel{\circ}{J}_{i_{0}}=\left(\alpha_{i_{0}}^{-}, \alpha_{i_{0}}^{+}\right)$, there exists a sequence $\left\{t_{n}\right\} \subset(0, T)$ such that $t_{n} \uparrow T$ and $b=\xi_{i_{0}}\left(t_{n}\right)$, that is, $u_{x}\left(b, t_{n}\right)=0 n \geq 1$. This contradicts (4.21). So we have (4.12), namely, (4.5) and (4.6). Thus the proof is complete.

\section{§5. The Case of a Bounded Domain}

Throughout this section, we assume (A1)-(A5) and consider the Dirichlet or Neumann problem (1.1)(1.2)(1.3ab). We assume further the following technical conditions on an initial data $u_{0}(x)$ :

(A6) $u_{0}(0)=u_{0}(L)=0$ and $u_{0}(x)>0$ for $x \in \Omega=(0, L)$;

(A7) there exist $a_{1}$ and $a_{2}$ in $\Omega=(0, L)$ such that $2 a_{1} \leq a_{2}, a_{1} \leq 2 a_{2}-L$, 
$\sigma_{a_{1}} u_{0}(x) \geq u_{0}(x)$ on $x \in\left[0, a_{1}\right]$ and $\sigma_{a_{2}} u_{0}(x) \geq u_{0}(x)$ on $x \in\left[a_{2}, L\right]$ where $\sigma_{a} u(x)$ $=u(2 a-x)$.

The main result in this section is as follows:

Theorem 5.1. Assume (A1)-(A7). Let $u(x, t)$ be a blow-up weak solution of problem (1.1)(1.2)(1.3a) or (1.1)(1.2) (1.3b) with blow-up time $t=T$ and, let $S\left(u_{0}\right)$ be the blow-up set of $u(x, t)$. Then

$$
S\left(u_{0}\right) \text { is a finite set, }
$$

and furthermore in the case of the Dirichlet problem,

$$
S\left(u_{0}\right) \subset\left(a_{1}, a_{2}\right) .
$$

First, we consider a weak solution $u(x, t)$ of the Dirichlet problem (1.1)(1.2)(1.3a). We need some lemmas.

Lemma 5.2. Assume (A1)-(A7). Let $u(x, t)$ be a blow-up weak solution of the Dirichlet problem (1.1)(1.2)(1.3a). Then

$$
\begin{array}{lll}
\sigma_{a_{1}} u(x, t) \geq u(x, t) & \text { for all } & (x, t) \in\left[0, a_{1}\right] \times[0, T) ; \\
\sigma_{a_{2}} u(x, t) \geq u(x, t) & \text { for all } & (x, t) \in\left[a_{2}, L\right] \times[0, T) .
\end{array}
$$

Proof. We show only (5.3). Set $v(x, t)=\sigma_{a_{1}} u(x, t)$. Then we see that $v(x, t)$ is also a weak solution of $(1.1)$ in $\left(0, a_{1}\right) \times(0, T)$. By the assumption (A7), we obtain that $v(x, 0)=\sigma_{a_{1}} u_{0}(x) \geq u_{0}(x)=u(x, 0)$. For boundary values of $u(x, t)$ in $\left(0, a_{1}\right) \times(0, T)$, we obtain that $v(0, t)=\sigma_{a_{1}} u(0, t) \geq 0=u(0, t)$ and $v\left(a_{1}, t\right)=u\left(a_{1}, t\right)$ for $t \in[0, T)$. Applying the comparison theorem to $v(x, t)$ and $u(x, t)$, we have that $v(x, t) \geq u(x, t)$ for $(x, t) \in\left(0, a_{1}\right) \times(0, T)$. This is a proof of (5.3).

This and Lemma 3.1 imply the following

Lemma 5.3. Let $u(x, t)$ be as in Lemma 5.2. Then

$$
\begin{aligned}
& u_{x}\left(a_{1}, t\right)>0 \quad \text { for all } t \in(0, T) \text {; } \\
& u_{x}\left(a_{2}, t\right)<0 \quad \text { for all } t \in(0, T) \text {. }
\end{aligned}
$$

Lemma 5.4. Let $u(x, t)$ be as in Lemma 5.3. Then for any $t_{0} \in(0, T)$, there exists $\delta=\delta\left(t_{0}\right)>0$ such that for any $a \in\left[a_{1}-\delta, a_{1}+\delta\right]$

$$
\sigma_{a} u(x, t) \geq u(x, t) \quad \text { for all }(x, t) \in[0, a] \times\left[t_{0}, T\right)
$$

and for any $a \in\left[a_{2}-\delta, a_{2}+\delta\right]$

$$
\sigma_{a} u(x, t) \geq u(x, t) \quad \text { for all }(x, t) \in[a, L] \times\left[t_{0}, T\right) .
$$

Proof. We only show (5.7). Noting the proof of Lemma 5.2, we see that it 
is enough to show the following: For any $t_{0} \in(0, T)$, there exists $\delta=\delta\left(t_{0}\right)>0$ such that for any $a \in\left[a_{1}-\delta, a_{1}+\delta\right]$,

$$
\sigma_{a} u\left(x, t_{0}\right)-u\left(x, t_{0}\right)>0 \quad \text { for all } x \in[0, a) .
$$

Therefore we show (5.9).

By (5.5), for any $t_{0} \in(0, T)$ there exists $\delta=\delta\left(t_{0}\right)>0$ such that

$$
u_{x}\left(x, t_{0}\right)>0 \quad \text { for }\left|x-a_{1}\right|<\delta .
$$

Set $h(a)=\min _{0 \leq x \leq a-\delta / 2}\left\{\sigma_{a} u\left(x, t_{0}\right)-u\left(x, t_{0}\right)\right\}$ for each $a \in[0, L / 2]$. Then, we see clearly that $h(a)$ is a continuous function in $[0, L / 2]$. Noting the proof of $(3.3)$, we obtain

$$
h\left(a_{1}\right)>0
$$

Hence there exists $\delta^{\prime}>0\left(0<\delta^{\prime} \leq \delta / 2\right)$ such that $h(a)>0$ for all $\left|a-a_{1}\right| \leq \delta^{\prime}$, namely

$$
\sigma_{a} u\left(x, t_{0}\right)-u\left(x, t_{0}\right)>0 \quad \text { for } 0 \leq x \leq a-\delta / 2,\left|a-a_{1}\right| \leq \delta^{\prime} .
$$

On the other hand, since $a-\delta / 2 \leq x \leq a$ and $\left|a-a_{1}\right| \leq \delta / 2$ imply $-\delta \leq$ $x-a_{1} \leq \delta / 2$, by $(5.10)$ we have

$$
\sigma_{a} u\left(x, t_{0}\right)-u\left(x, t_{0}\right)>0 \quad \text { for } a-\delta / 2 \leq x \leq a, \quad\left|a-a_{1}\right| \leq \delta^{\prime} .
$$

Combining (5.12) and (5.13), we obtain (5.9). The proof is complete.

This and Lemma 3.1 imply the following

Lemma 5.5. Let $u(x, t)$ be as in Lemma 5.4. Then,

$$
\begin{array}{lll}
u_{x}(x, t)>0 & \text { for all } & (x, t) \in\left[a_{1}-\delta, a_{1}+\delta\right] \times\left(t_{0}, T\right) \\
u_{x}(x, t)<0 & \text { for all } & (x, t) \in\left[a_{2}-\delta, a_{2}+\delta\right] \times\left(t_{0}, T\right) .
\end{array}
$$

We are now ready to prove Theorem 5.1 in the case of the Dirichlet problem.

Proof of Theorem 5.1 (the case of the Dirichlet problem). By Lemma 5.5 and Lemma 4.1, we see that $S\left(u_{0}\right) \cap\left(a_{1}-\delta, a_{2}+\delta\right)$ is a finite set. Hence, if we show that $\left[0, a_{1}-\delta\right] \cup\left[a_{2}+\delta, L\right] \subset[0, L] \backslash S\left(u_{0}\right)$, the proof is complete. We show only

$$
\left[0, a_{1}-\delta\right] \subset[0, L] \backslash S\left(u_{0}\right)
$$

Now we assume that $x_{0} \in\left[0, a_{1}-\delta\right]$ is a point of $S\left(u_{0}\right)$. Then, by (A7) we have that $2 a_{1}-x \in\left[a_{1}, a_{2}\right]$. It follows from Lemma 5.2 that $2 a_{1}-x_{0} \in S\left(u_{0}\right)$. Since $S\left(u_{0}\right) \cap\left(a_{1}-\delta, a_{2}+\delta\right)$ is a finite set, there exists $a_{3} \in\left(a_{1}-\delta, a_{1}+\delta\right)$ such 
that $2 a_{3}-x_{0} \notin S\left(u_{0}\right)$ and $0 \leq x_{0}<a_{3}$. By the fact that $\sigma_{a_{3}} u(x, t) \geq u(x, t)$ for $(x, t) \in\left[0, a_{3}\right] \times\left[t_{0}, T\right)$, we obtain that $x_{0} \notin S\left(u_{0}\right)$. This contradicts the assumption that $x_{0} \in S\left(u_{0}\right)$, and thus we prove (5.16).

Next we cosider a blow-up weak solution $u(x, t)$ of the Neumann problem (1.1)(1.2)(1.3b). We can consider the following three cases:

$$
u(0, t)=u(L, t)=0 \quad \text { for all } t \in[0, T) .
$$

(II) (i) $u(0, t)=0$ for all $t \in[0, T)$ and there exists $t_{1} \in(0, T)$ such that $u\left(L, t_{1}\right)>0$, $u\left(0, t_{1}\right)>0$.

(ii) $u(L, t)=0$ for all $t \in[0, T)$ and there exists $t_{1} \in(0, T)$ such that

(III) there exist $t_{1}, t_{2} \in(0, T)$ such that $u\left(0, t_{1}\right)>0$ and $u\left(L, t_{2}\right)>0$.

Proof of Theorem 5.1 (the case of the Neumann problem).

Case (I). Since $u(x, t)$ can be regarded as a solution of the Dirichlet problem, this case comes to the case of the Dirichlet problem.

Case (II). We only prove in case (ii). Extend a weak solution $u(x, t)$ as

$$
\tilde{u}=\left\{\begin{array}{lr}
u(x, t) & 0 \leq x \leq L \\
u(-x, t) & -L \leq x \leq 0 .
\end{array}\right.
$$

Then, we see that $\tilde{u}(x, t)$ is a weak solution of the Neumann problem and that $\tilde{u}( \pm L, t)=0$ for $t \geq t_{1}$ and $\tilde{u}\left(x, t_{1}\right)>0$ for $x \in(-L, L)$. Hence this case comes to the case (I).

Case (III). By extending $u(x, t)$ as (5.17) and appropriate rescaling of the variables, this problem can be converted into the form (1.1)(1.2) with $\Omega=S^{1}$ $=\mathbf{R} / \mathbf{Z}$. Set $t_{0}=\max \left\{t_{1}, t_{2}\right\}$. Then, it follows from the assumption (III) and Lemma 2.3 that $u(x, t)>0$ for all $(x, t) \in S^{1} \times\left(t_{0}, T\right)$ and $u(x, t)$ is a classical solution of (1.1) on $S^{1} \times\left(t_{0}, T\right)$. Noting Lemma 4.5 and Lemma 3.2 and using the same methods as Chen-Matano [4], we can prove the assertion in this case. The proof is complete.

Remark 5.6. Considering the proof in this section, we can also get the similar result as Theorem 5.1, even if we extend conditions (A6) and (A7) on $u_{0}(x)$ to the following condition:

(*) there exists a finite family $\left\{\Omega_{i}\right\}_{i=1}^{i=l}$ of open intervals such that $\bigcup_{i=1}^{l} \Omega_{i} \subset(0, L)$, $\Omega_{i} \cap \Omega_{j}=\phi(i \neq j)$ and $u_{0}(x)=0$ in $(0, L) \backslash \bigcup_{i=1}^{l} \Omega_{i}$, and such that (A6) and (A7) hold with $\Omega$ replaced by $\Omega_{i}$. 


\section{§6. Asymptotic Behavior of an Interface}

In this section, we consider the Cauchy problem (1.1)(1.2)(1.3c). We assume (A1)-(A4) and assume the following conditions on an initial data $u_{0}(x)$ :

$$
u_{0}(x)>0, x \in(0, L) \text { and } u_{0}(x)=0, x \in \mathbf{R} \backslash(0, L) .
$$

Furthermore, we assume the following conditions for the finite propagation of the interface of a weak solution $u(x, t)$ to $(1.1)(1.2)(1.3 \mathrm{c})$ in $t \in(0, T)$ :

$$
\int_{0}^{1} \frac{d \eta}{b(\eta)}<\infty
$$

$$
f(0)=0
$$

(A11) there exists a $C^{1}$-function $G(u)$ on $[0, \infty)$ such that

$$
G(u) \geq f(u) \text { and } G^{\prime} b-G b^{\prime} \geq 0 \text { for all } u \geq 0 .
$$

Now we can show a finite propagation of an interface of a weak solution.

Theorem 6.1 (a finite propagation of an interface). Assume (A1)-(A4) and (A8)-(A11). Let $u(x, t)$ be a blow-up weak solution of $(1.1)(1.2)(1.3 \mathrm{c})$ with blow-up time $t=T . \quad$ Then, there exist continuous functions $\xi_{i}(t):[0, T) \rightarrow \mathbf{R}(i=1,2)$ such that

$$
\left(\xi_{1}(t), \xi_{2}(t)\right)=\{x \in \mathbf{R} \mid u(x, t)>0\} \quad \text { for each } t \in[0, T),
$$

$\xi_{1}(t)$ is a monotone decreasing function and $\xi_{2}(t)$ is a monotone increasing function, and furthermore,

$$
-\infty<\xi_{1}(t)<\xi_{2}(t)<\infty \quad \text { for each } t \in[0, T) .
$$

Next we state behavior of the interface of $u(x, t)$ near the blow-up time $t=T$. For this aim, we further assume (A5) or the condition

$$
\lim _{u \rightarrow \infty} \frac{H(u)}{u^{2}}=0
$$

where $H(u)=\int_{0}^{u} f(\xi) d \xi$

Theorem 6.2. Let $S\left(u_{0}\right)$ be the blow-up set of $u(x, t)$ where $u(x, t)$ is as in Theorem 6.1 and let $\xi_{i}(t)(i=1,2)$ is the interface of $u(x, t)$. Then the following two results hold.

(i) If (A5) hods, then

$$
S\left(u_{0}\right) \subset[0, L]
$$

and 


$$
-\infty<\lim _{t \uparrow T} \xi_{1}(t)<\lim _{t \uparrow T} \xi_{2}(t)<\infty,
$$

and furthermore if (A7) holds, then

$$
S\left(u_{0}\right) \subset\left(a_{1}, a_{2}\right) \text { and } S\left(u_{0}\right) \text { is a finite set. }
$$

(ii) If (A12) holds,

$$
S\left(u_{0}\right)=\mathbf{R} \cup\{\infty\} \cup\{-\infty\}
$$

and

$$
\lim _{t \uparrow T} \xi_{1}(t)=-\infty \text { and } \lim _{t \uparrow T} \xi_{2}(t)=\infty .
$$

Remark 6.3. If $p>1$ and $m>1$, equation (1.4) satisfies these conditions (A9)-(A11) with $G(u)=f(u)=u^{p / m}$. If $p / m<1$, equation (1.4) satisfies condition (A12).

First we prove Theorem 6.1. We need the following lemma:

Lemma 6.4. Assume (A1)-(A4) and (A9)-(A11). Let $u(x, t)$ be a weak solution of (1.1)(1.2)(1.3c). Suppose that there exist $\left(a, t_{1}\right) \in \mathbf{R} \times[0, T)$ and $M$ $>0$ such that

$$
\begin{array}{ll}
u\left(x, t_{1}\right)=0 & \text { for } \quad x \geq a ; \\
u(a, t) \leq M & \text { for } \quad t \in\left[t_{1}, T\right) .
\end{array}
$$

Then, there exist $l>0$ and $h>0$ depending on only $M$ such that

$$
u(x, t)=0 \quad \text { for } \quad(x, t) \in[a+l, \infty) \times\left[t_{1}, t_{1}+h\right] \cap\left[t_{1}, T\right) .
$$

Proof. Consider the function $\psi:[0, \infty) \rightarrow[0, \infty)$ such that

$$
\psi(u)=\int_{0}^{u} \frac{d \eta}{b(\eta)}(\text { see Knerr }[11]) .
$$

Then, by (A1) and (A9) we see that $\psi(u)$ is well defined and is an onto and one to one mapping. Put

$$
v(x, t)=\psi(u(x, t))
$$

Since $u(x, t)$ satisfies the equation (1.1) in $(x, t)$-set where $u(x, t)>0$, a simple computation gives

$$
v_{t}=\frac{1}{b^{\prime}\left(\psi^{-1}(v)\right)} v_{x x}+\left(v_{x}\right)^{2}+\frac{f\left(\psi^{-1}(v)\right)}{b\left(\psi^{-1}(v)\right) b^{\prime}\left(\psi^{-1}(v)\right)}
$$

where $\psi^{-1}(v)$ is the inverse function of $v=\psi(u)$. 
Let us consider the following function $v(x, t)$ :

$$
v(x, t)=(\eta(t)-C(x-a))^{+} \quad \text { for } \quad x \geq a, t \geq t_{1}
$$

where $\eta(t)$ satisfies the problem

$$
\left\{\begin{array}{l}
\eta\left(t_{1}\right)=\eta_{1}=\psi(M)>0 \\
\eta_{t}=C^{2}+\frac{G\left(\psi^{-1}(\eta)\right)}{b\left(\psi^{-1}(\eta)\right) b^{\prime}\left(\psi^{-1}(\eta)\right)}
\end{array}\right.
$$

and $C$ is a positive constant. Then $\eta(t)$ is represented as the following:

$$
\eta(t)=\bar{G}^{-1}\left(\bar{G}\left(\eta_{1}\right)-\left(t-t_{1}\right)\right)
$$

where

$$
\bar{G}(\eta)=\int_{\eta}^{\infty} \frac{1}{C^{2}+\frac{G\left(\psi^{-1}(\eta)\right)}{b\left(\psi^{-1}(\eta)\right) b^{\prime}\left(\psi^{-1}(\eta)\right)}} d \eta
$$

Here we note that

$$
\bar{G} \leq \int_{\eta}^{\infty} \frac{b\left(\psi^{-1}(\eta)\right) b^{\prime}\left(\psi^{-1}(\eta)\right)}{G\left(\psi^{-1}(\eta)\right)} d \eta=\int_{\psi^{-1}(\eta)}^{\infty} \frac{b^{\prime}(\xi)}{f(\xi)} d \xi<\infty\left(\psi^{\prime}=\frac{1}{b}\right)
$$

and $\xi=\bar{G}^{-1}(\eta)$ is the inverse function of $\eta=\bar{G}(\xi)$. And we have

$$
\eta(t)<\infty, \quad t \in\left[t_{1}, t_{1}+\bar{h}\right) \text { and } \eta(t) \uparrow \infty \text { as } t \uparrow t_{1}+\bar{h},
$$

where $\bar{h}=\bar{G}\left(\eta_{1}\right)$. Since $v(x, t)=\eta(t)-C(x-a)$ where $\eta(t)>C(x-a)$, we obtain

$$
v_{t}=\frac{1}{b^{\prime}\left(\psi^{-1}(v)\right)} v_{x x}+\left(v_{x}\right)^{2}+\frac{G\left(\psi^{-1}(\eta)\right)}{b\left(\psi^{-1}(\eta)\right) b^{\prime}\left(\psi^{-1}(\eta)\right)}
$$

Considering that $\frac{G(\xi)}{b(\xi) b^{\prime}(\xi)}$ is a monotone increasing function by (A1) and (A11), and that $v(x, t)=(\eta(t)-C(x-a))^{+} \leq \eta(t)$ for $x \geq a$, namely $\psi^{-1}(v) \leq \psi^{-1}(\eta)$ for $x \geq a$, we have

$$
v_{t} \geq \frac{1}{b^{\prime}\left(\psi^{-1}(v)\right)} v_{x x}+\left(v_{x}\right)^{2}+\frac{G\left(\psi^{-1}(v)\right)}{b\left(\psi^{-1}(v)\right) b^{\prime}\left(\psi^{-1}(v)\right)} .
$$

Hence, if we put $w(x, t)=\psi^{-1}(v(x, t))$, we obtain

$$
b(w)_{t} \geq w_{x x}+f(w), \quad t \in\left[t_{1}, t_{1}+\bar{h}\right), \eta(t)>C(x-a), x \geq a .
$$

On the other hand, since $v(x, t)=0$ namely $w(x, t)=0$ in $(x, t)$-set where $x \geq a$, 
$t \in\left[t_{1}, t_{1}+\bar{h}\right)$ and $\eta(t)<C(x-a)$, and since $f(0)=0$, we have

$$
b(w)_{t}=w_{x x}+f(w), \quad t \in\left[t_{1}, t_{1}+\bar{h}\right), \eta(t)<C(x-a), x \geq a .
$$

Since $w=0$ on $\eta(t)=C(x-a)$ and $b(0)=0$, we have

$$
w_{x}=\left.v_{x} b(w)\right|_{\eta=C(x-a)}=0 .
$$

Hence, combining (6.21), (6.22) and (6.23), we see that $w(x, t)$ is a super-solution of $(1.1)$ in $[a, \infty) \times\left[t_{1}, t_{1}+\bar{h}\right)$.

On the other hand, by (6.9) and (6.10) we have

$$
w(a, t)=\psi^{-1}(v(a, t)) \geq \psi^{-1}(\eta(t)) \geq \psi^{-1}(\psi(M))=M \geq u(a, t)
$$

for $t \in\left[t_{1}, t_{1}+\bar{h}\right) \cap\left[t_{1}, T\right)$ and

$$
w\left(x, t_{1}\right) \geq 0=u\left(x, t_{1}\right) \quad \text { for } \quad x \geq a .
$$

Applying the comparison theorem to $w(x, t)$ and $u(x, t)$ on $[a, \infty) \times\left[t_{1}, t_{0}\right)$ where $t_{0}=\min \left\{t_{1}+\bar{h}, T\right\}$, we obtain

$$
w(x, t) \geq u(x, t) \quad \text { for } \quad(x, t) \in[a, \infty) \times\left[t_{1}, t_{0}\right) .
$$

Namely,

$$
u(x, t)=0 \quad \text { for } \quad x \geq a, \eta(t)<C(x-a), t \in\left[t_{1}, t_{0}\right) .
$$

If we choose $h=\bar{h} / 2$ and $l=\eta\left(t_{1}+h\right) / C$, we can show the assertions of Lemma 6.3 .

Proof of Theorem 6.1. Set $\xi_{1}(t)=\inf \{x \mid u(x, t)>0\}$ and $\xi_{2}(t)=\sup \{x \mid u(x, t)$ $>0\}$. Then, by Lemma 2.2 and Lemma 2.3 it is obvious that $\xi_{1}(t)$ is a monotone decreasing function and $\xi_{2}(t)$ is a monotone increasing and that (6.2) holds (cf. Knerr [11]).

Next, we show (6.3) and here we only prove

$$
\xi_{2}(t)<\infty \quad \text { for each } t \in(0, T) .
$$

Assume that (6.28) does not hold. Then, it follows that

$$
\xi_{2}(t)=\infty \quad \text { for all } t \in(0, T)
$$

or there exists $t_{0} \in(0, T)$ such that

$$
\xi_{2}(t)<\infty \text { for } t \in\left(0, t_{0}\right) \text { and } \xi_{2}\left(t_{0}\right)=\infty .
$$

We only drive a contradiction in case of (6.30). In case of (6.29), we can also drive it similarly.

Set

$$
M=\sup \left\{u(x, t) \mid(x, t) \in \mathbf{R} \times\left[0, t_{0}+\delta\right]\right\}
$$


where $0<\delta<T-t_{0}$. Then, by Lemma 6.3 there exist $l>0$ and $h>0$ such that (6.11) holds with $T$ replaced by $t_{0}+\delta$. Choose $a>0$ and $t_{1} \in\left(0, t_{0}\right)$ such that $t_{0}<t_{1}+h$ and $a=\xi_{2}\left(t_{1}\right)$. Then, we obtain

$$
u(x, t)=0 \text { for }(x, t) \in[a+l, \infty) \times\left[t_{1}, t_{1}+h\right] \cap\left[t_{1}, t_{0}+\delta\right),
$$

that is,

$$
\xi_{2}\left(t_{0}\right) \leq a+l
$$

This contradicts (6.30) and thus (6.28) is shown.

Finally we can show the continuity of $\xi_{i}(t)$ by similar methods as that show (6.28), and we omit this proof.

We need some lemmas to show Theorem 6.2.

Lemma 6.5. Assume (A1)-(A4) and (A8). Let $u(x, t)$ be a weak solution of (1.1)(1.2)(1.3c) and let $a \in \mathbb{R} \backslash(0, L)$. Then

$$
\sigma_{a} u(x, t) \geq u(x, t), \begin{array}{ll}
\text { for all } \quad x \in[a, \infty) & \text { if } a \geq L \\
\text { for all } \quad x \in(-\infty, a] & \text { if } a \leq 0
\end{array}, \quad t \in(0, T) .
$$

Therefore, $u(x, t)$ is a monotone decreasing function on $x \geq L$ and a monotone increasing function on $x \leq 0$ for each $t \in(0, T)$.

Proof. This proof is similar to it of Lemma 5.2. We omit it.

Lemma 6.6. Let $\xi_{i}(t) \quad(i=1,2)$ be as in Theorem 6.1. Then

$$
\lim _{t \uparrow T} \xi_{1}(t)=-\infty \quad \text { if and only if } \lim _{t \uparrow T} \xi_{2}(t)=\infty .
$$

Proof. This proof is obvious by Lemma 6.5.

Proof of Theorem 6.2 (i). First, we show that $S\left(u_{0}\right) \subset[0, L]$.

Assume that $x_{0} \in S\left(u_{0}\right)$ is not a point in $[0, L]$. Without loss of generality, we may assume that $x_{0}>L$. By Lemma 6.5 , we have that $\left[L, x_{0}\right) \subset S\left(u_{0}\right)$. It follows from Lemma 3.1 and Lemma 6.5 that for any $\delta>0$ small enough there exists $t_{0}=t_{0}(\delta) \in(0, T)$ such that $u(x, t)>0$ for $(x, t) \in\left[L, x_{0}-\delta\right] \times\left[t_{0}, T\right)$ and $u_{x}(x, t)>0$ in $(x, t) \in\left[L, x_{0}-\delta\right] \cup\left[t_{0}, T\right)$. Using Lemma 3.3, we have that $\left(L, x_{0}-\delta\right) \subset S\left(u_{0}\right)^{c}$. This contradicts that $\left[L, x_{0}\right) \subset S\left(u_{0}\right)$ and thus we show that $S\left(u_{0}\right) \subset[0, L]$.

Hence, noting that $u(x, t) \leq M(\delta)$ for $(x, t) \in(-\infty,-\delta] \cup[L+\delta, \infty)$ $\times[0, T)(\delta>0)$ and using the similar method as in the proof of Theorem 6.1 , we see (6.5).

Next we further assume (A7) and prove (6.6). Consider $u(x, t)$ and $\sigma_{a_{2}} u(x, t)$ in $\left(a_{2}, \infty\right) \times(0, T)$. By the comparison theroem on a half space, we have that $\sigma_{a_{2}} u(x, t) \geq u(x, t)$ in $\left(a_{2}, \infty\right) \times(0, T)$ (cf. proof of Lemma 5.2). Using Lemma 
3.1, we obtain that $u_{x}\left(a_{2}, t\right)<0$ for $t \in(0, T)$. Hence, by the similar method as in the proof of Lemma 5.4 and by the continuity of $\xi_{2}(t)$, we have that, if $t_{1} \in(0, T)$ is sufficiently close to $t=0$, then $\sigma_{a} u\left(x, t_{1}\right) \geq u\left(x, t_{1}\right)$ in $[a, \infty)$ $\times\left[t_{1}, T\right)$ where $\left|a-a_{2}\right| \leq \delta$ for some $\delta>0$. Using Lemma 3.1 again, we obtain that $u_{x}(a, t)<0$ for $\left|a-a_{2}\right|<\delta, t_{1}<t<T$. Also similarly we obtain that $u_{x}(a, t)>0$ for $\left|a-a_{1}\right|<\delta^{\prime}, t_{1}<t<T$ for some $\delta^{\prime}>0$. Therefore as we prove Theorem 5.1, we obtain (6.6). The proof is complete.

Next we prove Theorem 6.2 (ii). We need the following lemma due to Imai-Mochizki [8].

Lemma 6.7. Assume (A1)-(A4) and (A12). Let $u(x, t)$ be a weak solution of the Dirichlet problem (1.1)(1.2)(1.3a). Then, $u(x, t)$ exists globally in time and stays bounded (in $\left.L^{\infty}(0, L)\right)$ as $t \uparrow \infty$.

Proof of Theorem 6.2 (ii). First, we show

$$
(-\infty, 0] \cup(L, \infty) \subset S\left(u_{0}\right) .
$$

Here, we only show that $[L, \infty) \subset S\left(u_{0}\right)$.

Assume that $x_{0} \in[L, \infty)$ is not a point in $S\left(u_{0}\right)$. Then, by Lemma 6.5 we have that for some $M_{1}>0$

$$
u(x, t) \leq M_{1} \quad \text { for } \quad(x, t) \in\left[x_{0}, \infty\right) \times[0, T),
$$

and by (A8) we see

$$
u(x, 0)=u_{0}(x)=0 \quad \text { for } \quad x \geq x_{0} .
$$

As we prove Theorem 6.1, we obtain

$$
\lim _{t \uparrow T} \xi_{2}(t)<\infty .
$$

Using Lemma 6.6, we have

$$
\lim _{t \uparrow T} \xi_{1}(t)>-\infty
$$

Choosing $x_{1}<\lim _{t \uparrow T} \xi_{1}(t)$ and $\lim _{t \uparrow T} \xi_{2}(t)<x_{2}$, we see that $u\left(x_{1}, t\right)=0$ and $u\left(x_{2}, t\right)$ $=0$ for $t \in(0, T)$. Since we can look $u(x, t)$ as a solution of the Dirichlet problem with $\Omega=\left(x_{1}, x_{2}\right)$, we see that $u(x, t)$ does not blow up at $t=T$ by Lemma 6.7. This is a contradiction since $t=T$ is assumed to be the blow-up time of $u(x, t)$ and thus we prove (6.33). Noting Lemma 6.5, we obtain that $(0, L) \subset S\left(u_{0}\right)$. Hence we see that $S\left(u_{0}\right)=\mathbf{R} \cup\{\infty\} \cup\{-\infty\}$ and that $\lim _{t \uparrow T} \xi_{2}(t)$ $=\infty$ and $\lim _{t \uparrow T} \xi_{1}(t)=-\infty$. The proof is complete. 


\section{References}

[1] Angenent, S., The zeroset of a solution of a parabolic equation, J. Reine Angew. Math., 390 (1988), 79-96.

[2] Aronson, D. G., Crandall, M. G. and Peletier, L. A., ' Stabilization of solutions of a degenerate nonlinear diffusion problem, Nonlinear Anal., 6(1982), 1001-1022.

[3] Bertsch, M., Kersner, R. and Peletier L. A., Positivity versus localization in degenerate diffusion equations, Nonlinear Anal., 9 (1985), 987-1008.

[4] Chen, X. -Y. and Matano, H., Convergence, asymptotic periodicity and finite-point blow-up in one-dimensional semilinear heat equations, J. Differential Equations, 78(1989), 160-190.

[5] Friedman, A. and McLeod, B., Blow-up of positive solutions of semilinear heat equations, Indiana Univ. Math. J., 34 (1985), 425-447.

[6] Galaktinov, V. A., Kurdyumov, S. P., Mikhailov, A. P. and Samrskii A. A., On unbounded solutions of the Cauchy problem for the parabolic equation $u_{t}=\nabla\left(u^{\sigma} \nabla u\right)$ $+u^{\beta}, \quad$ Dokl. Akad.Nauk SSSR., 252 (1980), 1362-1364; Soviet Phys. Dokl., 25(1980), 458459.

[7] Galaktinov, V. A., Proof of the localization of unbounded solutions of the non-linear partial differential equation $u_{t}=\left(u^{\sigma} u_{x}\right)_{x}+u^{\beta}$, Differential'nye Uravneniya, 21 (1985), 15-23; Differential Equations, 21 (1985), 11-18.

[8] Imai, T. and Mochizuki, K., On blow-up Solutions for quasilinear degenerate parabolic equations, preprint 1989.

[9] Itaya, N., A note on the blowup-nonblowup problems in nonlinear parabolic equations, Proc. Japan Acad., 55(1979), 241-244.

[10] _ On some subjects related to the blowing-up problem in nonlinear parabolic equations, Lecture Notes in Num. Apll. Anal., 2 (1980), 27-38, Kinokuniya.

[11] Knerr, B. F., The porous medium equation in one dimension, Trans. Amer. Math. Soc., 234 (1977), 381-415.

[12] Ladyzenskaja, O. A., Solonnikov, V. A. and Ural'ceva, N. N., Linear and Quasilinear Equations of Parabolic Type, Trncl. Math. Monographs, 23 AMS Providence, R. I. 1968.

[13] Oleinik, O. A., Kalashnikov A. S. and Chzou Yui-Lin, The Cauchy problem and boundary problems for equations of the type of nonlinear filtration, Izv. Akad.Nauk SSSR Ser.Math., 22 (1958), 667-704 (Russian). 